\title{
Prosodie et morphosyntaxe : un lien à questionner pour l'enseignement du FLE
}

Prosody and morphosyntax: a significant link relevant for teaching French as a foreign language

\section{Marie-Françoise Bourvon}

\section{OpenEdition}

Journals

Édition électronique

URL : http://journals.openedition.org/tipa/1296

DOI : $10.4000 /$ tipa. 1296

ISSN : 2264-7082

Éditeur

Laboratoire Parole et Langage

Référence électronique

Marie-Françoise Bourvon, « Prosodie et morphosyntaxe : un lien à questionner pour l'enseignement du FLE », TIPA. Travaux interdisciplinaires sur la parole et le langage [En ligne], 30 | 2014, mis en ligne le 17 décembre 2014, consulté le 19 avril 2019. URL : http://journals.openedition.org/tipa/1296 ; DOI : 10.4000/tipa.1296

Ce document a été généré automatiquement le 19 avril 2019.

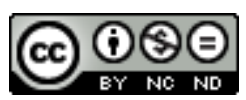

La revue TIPA. Travaux interdisciplinaires sur la parole et le langage est mise à disposition selon les termes de la licence Creative Commons Attribution - Pas d'Utilisation Commerciale - Pas de Modification 4.0 International. 


\section{Prosodie et morphosyntaxe : un lien à questionner pour l'enseignement du FLE}

Prosody and morphosyntax: a significant link relevant for teaching French as a foreign language

Marie-Françoise Bourvon

Nous remercions vivement l'équipe LIDILE, et tout particulièrement Najib Arbach de nous avoir confié son corpus et Saandia Ali de nous avoir guidée dans l'utilisation de Praat.

\section{Introduction}

1 «Accentuation» et «intonation» sont les éléments communs aux définitions de la prosodie données par exemple par Carton (1974), Gadet (1989), Léon (1992), Astésano (2001), ainsi qu'à celles que donnent, dans une perspective didactique, Lauret (2007) et Intravaia (2000) - à la différence près que ce dernier préfère au terme d'« accentuation » celui d'« accent».

En français, l'accent dit tonique fait de la syllabe qui le porte une syllabe proéminente qui est une proéminence primordiale, et en particulier pour l'enseignement-apprentissage du FLE. Cet accent est caractérisé par un allongement de la syllabe pleine de fin de mot ou de groupe, et parmi les différents paramètres acoustiques que sont la durée, la fréquence fondamentale et l'intensité et qui servent à décrire l'accent en français, la durée est donc l'élément le plus important.

Le fait que cet accent ne soit pas systématiquement perçu par les locuteurs et auditeurs francophones (Carton, 1974; Wioland, 1991) ne suffit pas à nier son existence. En revanche, le fait que l'accent entretienne des relations étroites avec la morphosyntaxe le signale comme essentiel dans l'élaboration d'énoncés oraux par les apprenants d'une L2. En effet, la construction syntaxique, les frontières entre les mots, les syntagmes, les 
propositions, sont matérialisées à l'oral par des groupes rythmiques, marqués par l'accent de groupe et les pauses de segmentation (Léon M. \& Léon P. 2002 ; Vaissière, 2010).

A partir d'une base de données $\mathrm{CIL}^{1}$ sous $\mathrm{CLAN}^{2}$, constituée d'interviews de locuteurs de français L2, cet article se propose de mettre en évidence et en relation erreurs prosodiques et morphosyntaxiques. L'analyse, qui n'est pas contrastive et n'envisage donc pas les interférences entre langue source et langue cible, mettra en corrélation l'absence d'accentuation de la syllabe de fin de groupe et/ou une segmentation erronée de la chaîne parlée, et les erreurs morphosyntaxiques.

Seront tout d'abord indiquées les raisons théoriques pour lesquelles les phénomènes étudiés privilégient l'accent de fin de groupe rythmique et la pause de segmentation, puis après quelques précisions sur le protocole expérimental adopté, seront présentés les résultats de l'analyse. Les premiers constats montrent d'une part que des échanges tels que les salutations et les reformulations de questions révèlent une utilisation différente des marques prosodiques par les natifs et les non natifs. Ils prouvent qu'erreurs morphosyntaxiques et prosodiques tissent bien des liens, et il sera question du déterminant nominal puis $\mathrm{du}$ " que » de subordination. Le corpus révèle aussi comment et à quelles conditions l'énumération et la répétition sont concomitantes de la prosodie attendue. S'appuyant sur cette analyse, la conclusion envisagera quelques pistes didactiques.

\section{Cadre théorique}

\subsection{L'accent tonique comme proéminence essentielle}

Parmi les différents éléments qui constituent la prosodie, et en particulier l'accentuation et l'intonation, il apparaît que l'accent dit tonique est une proéminence de l'oral particulièrement caractéristique du français. Pour Wioland (1983), parmi les différents paramètres acoustiques qui servent à décrire cet accent, à savoir la durée, la fréquence fondamentale et l'intensité, la durée est l'élément le plus important. Il rejoint ainsi Delattre (1966 : 70) pour qui la durée est celui des trois éléments acoustiques qui est le plus étroitement uni à l'accent et [...] le seul des trois qui en soit toujours un facteur. La durée est un élément essentiel parce qu'il ne peut être remis en question, il l'est aussi dans la perspective didactique qui est la nôtre parce qu'il peut être expliqué, montré, dans la réalité de la classe, sans matériel ni technologie particulière. En effet, l'allongement de la syllabe accentuée est le paramètre, sinon le plus immédiatement perceptible à l'oreille, du moins le plus à même d'être signalé, en perception, à des apprenants ; le plus à même aussi d'être travaillé en production. D'autre part, puisque durée, intensité et fréquence fondamentale sont le plus souvent liées, le fait de donner priorité à la durée n'équivaut pas à la non prise en compte des autres paramètres.

6 Un autre accent, emphatique ou secondaire (par ex. Pasdeloup, 1990 ; Astésano, 2001) permet au locuteur d'insister sur un mot de son choix. Cet accent touche une autre syllabe que celle qui porte habituellement l'accent, le plus souvent la première mais aussi parfois la deuxième syllabe du mot. La distinction est faite entre un accent « intellectuel » ou « didactique » comme dans :

«Ce mot n'est pas "variable mais "invariable ! ${ }^{3}$ 
«C'est é"pouvantable!»

8 Cet accent secondaire, emphatique, est souvent accompagné d'un détachement des syllabes qui l'encadrent:

« Il ne s'agit pas de \#"sus\#pendre mais de \#"su\#pprimer l'écotaxe! »

9 Cet accent est le résultat d'un choix du locuteur et non pas d'une contrainte morphosyntaxique (Astésano, 2001 : 63). D'autre part, il se caractérise, autant que par un allongement, par un renforcement de la «tension" initiale qui existe dans la parole neutre (Vaissière, 2010), par la hauteur et les modifications du patron de la fréquence fondamentale (Séguinot, 1976; Dahan \& Bernard, 1996, cités par Astésano, 2001) : il n'en sera pas ici question.

\subsection{L'accent tonique comme marque de fin de groupe}

10 L'unité prosodique concernée par l'accent est la syllabe. Dans un énoncé, les syllabes ne sont pas toutes produites avec la même intensité, certaines sont proéminentes, accentuées, d'autres inaccentuées ou moins accentuées. Pour ce qui est du paramètre que nous privilégions, la durée, on admet qu'une syllabe accentuée, en français standard, est en moyenne deux fois plus longue qu'une syllabe non accentuée (Delattre, 1962 ; Wenk \& Wioland, 1982 ; Léon, 1992).

11 Cet accent a une valeur démarcative et non distinctive. En effet, l'accent tonique en français n'est pas un accent de mot et n'a donc pas de fonction distinctive, comme en espagnol par exemple, où número numero, numeró, respectivement ['numero], [nu'mero] et [nume'ro] ont comme équivalents français : un numéro, je numérote et il numérota. Cette fonction distinctive existe dans de nombreuses langues: en plus de l'espagnol et de l'anglais, on peut aussi citer le russe. Par ailleurs on peut remarquer que le français n'est pas non plus une langue à accent fixe, contrairement par exemple au finnois et au tchèque qui ont un accent de mot affectant toujours la dernière syllabe, ou au polonais où l'accent porte toujours sur l'avant-dernière syllabe.

12 En français, c'est la dernière syllabe prononcée d'un groupe de mots qui porte l'accent, et non la dernière syllabe du mot, sauf bien sûr si le groupe ne comporte qu'un mot. Le mot «bonjour", accentué dans «bonjour» seul ([bo'zuR]), perd son accent au profit de « madame » dans l'énoncé « Bonjour Madame » ([GozuRma'dam]). Ce qui fait de « Bonjour Madame » une unité prosodique composée de deux mots graphiques.

L'unité prosodique supérieure à la syllabe est donc le groupe, articulé et perçu comme une succession de syllabes. La dernière syllabe de ce groupe, marquée par l'accent, est très généralement suivie d'une pause.

\subsection{Le groupe accentuel comme syntagme prosodique}

13 Ce groupe porte, en fonction des perspectives dans lesquelles il est étudié, des appellations multiples, et l'on parle, entre autres et selon les auteurs, de groupe de sens (Vaissière, 2010), sémantique (Léon M. \& Léon P. 2002), accentuel (Carton, 1974 ; Gadet, 1989 ; Martin, 2008), rythmique (Léon, 2001), phonétique (Malmerg, 1954). Une revue rapide 
de la façon dont ce groupe est décrit permet de mettre en évidence quelques caractéristiques de cette notion très discutée.

Carton (1974 : 102-103), envisageant la façon de définir ce groupe conclut ainsi :

Pratiquement, ne pourrait-on enseigner qu'il y a accent, marquant une fin de groupe accentuel, chaque fois qu'on pourrait faire une pause sans nuire ni au sens ni à la grammaire? Ainsi, dans la phrase : «L'ouverture / de la bouche / fait justement / comme un petit rond / qui représente / un o » : il n'y a que six accents possibles, quand l'expressivité est minimale. Il semble qu'on puisse définir grammaticalement et sémantiquement à la fois, des groupes accentuels virtuels, c'est-à-dire susceptibles dans certains contextes, de porter l'accent.

15 Un peu plus loin il ajoute:

Si beaucoup de francophones ont l'impression que leur langue est «sans accent tonique ", c'est surtout parce que l'unité accentuelle est élastique ; mais l'appui mutuel du sens et de la syntaxe peut permettre de définir des unités accentuelles virtuelles.

Pour Martin un «mot prosodique », unité minimale prosodique, est constitué par une séquence ne comportant qu'une seule syllabe proéminente, que cette proéminence corresponde à un accent lexical (tonique) ou un accent secondaire (c'est-à-dire d'insistance) : le «mot prosodique » correspond donc au groupe accentuel et ne contient qu'une seule syllabe proéminente. Martin (2008:14-15) rejette la validité de l'affirmation selon laquelle le groupe accentuel correspondrait au groupe de sens.

Cette notion [explique-t-il] pose [...]des difficultés lorsque l'on compare des phrases telles que "L'ami de Max» et "L'hippopotame de Nabuchodonosor », et que la première ne présente en général qu'un seul accent lexical sur «Max» (et éventuellement un autre sur la première syllabe de " ami »), alors que la seconde en révèle (presque) obligatoirement deux, soit sur les syllabes finales de " hippopo tame " et de «Nabuchodonosor » soit sur la première syllabe de «hippopotame» et sur la finale de "Nabuchodonosor. "

17 Martin propose donc de considérer qu'il existe des groupes accentuables (et non plus nécessairement accentués) dans lesquels les mots sont unis par des liens de dépendance grammaticale, et que c'est un paramètre rythmique qui déterminera alors l'accentuation effective. Ces groupes rythmiques comportent en moyenne 2,5 syllabes, le plus souvent trois ou quatre et très rarement plus de six syllabes (Fónagy, 1980; Wenk \& Wioland,1982 ; Wioland, 1983 ; Astésano, 2001).

Delais-Roussarie (2000: 102) va dans le même sens. Pour elle, les locuteurs segmentent prosodiquement un énoncé de manière à faire ressortir sa structure morpho-syntaxique et à respecter des principes rythmiques fondamentaux. Elle explique (Delais-Roussarie, 2011: 37) que la frontière droite du groupe accentuel est marquée par la présence d'un accent final sur sa dernière syllabe et précise :

En identifiant dans le corpus Anita Musso les syllabes recevant un accent final, il a été aisé de construire des groupes accentuels et de les comparer aux syntagmes phonologiques définis sur bases syntaxiques. Il est alors apparu clairement que les frontières des groupes accentuels correspondent aux frontières de syntagmes phonologiques dans plus de $85 \%$ des cas.

19 Vaissière (2010) quant à elle, liste les points faisant consensus : l'idée selon laquelle le groupe de sens ou unité équivalente comporte au moins un mot plein (lexical) et son (ou ses) mot(s) outil(s) et qu'il peut comporter plusieurs mots pleins liés par le sens; le fait que les locuteurs ont tendance à équilibrer la longueur des groupes de sens successifs et que surtout le nombre de groupes est susceptible de varier en fonction du contexte par exemple. 
20 L'ensemble de ces définitions met bien en évidence le lien entre prosodie et morphosyntaxe.

Par ailleurs, dans une perspective d'enseignement, qui est aussi la nôtre, la définition de Carton (1974) associe accent et pause. C'est ce que l'on retrouve, dans la même perspective, d'enseignement du FLE cette fois, chez Intravaia (2000:173): [...] le rythme français assume une importante fonction démarcative (segmentatrice, syntaxique). En effet, les accents et les pauses délimitent les unités de sens et conditionnent la segmentation de la phrase en groupes sémantico-syntaxiques.

\section{Protocole expérimental}

\subsection{Hypothèse}

21 L'accent dit tonique en tant que proéminence de l'oral se caractérise par le fait qu'il touche la syllabe de fin de groupe, les frontières de ce groupe étant aussi marquées par des pauses qui assurent la segmentation du continuum sonore. Puisque le lien entre ces marques prosodiques et la morphosyntaxe n'est plus à démontrer, nous posons que la prosodie d'un locuteur francophone non natif est révélatrice de son degré de maîtrise de la langue.

Dans un corpus d'interlangue, nous nous proposons donc d'analyser deux des proéminences liées au découpage de la parole en groupes : l'allongement de la syllabe accentuée et la pause de segmentation, la question étant de savoir si ou dans quelle mesure les erreurs prosodiques sont concomitantes et symptomatiques d'erreurs morphosyntaxiques.

\subsection{Corpus}

Le corpus analysé est composé de quarante entretiens enregistrés. Il s'agit en réalité d'un sous-corpus, le corpus total étant constitué pour chaque locuteur-trice non natif-ve d'un entretien de 20 à $30 \mathrm{mn}$, d'une lecture de $3 \mathrm{mn}$ et d'une production écrite d'une page A4. Les entretiens ont été menés pour 25 d'entre eux par des locuteurs natifs et pour les 15 autres par des locuteurs non natifs de niveau C2. Les interviewés, douze arabophones, huit anglophones, treize hispanophones et sept sinophones, sont de niveau B2 ou C1 du CECRL. Les interviewés sont soit en situation d'apprentissage et inscrits dans un DU de langue française au CIREFE ${ }^{4}$, soit insérés dans la vie professionnelle (en France ou à l'étranger) et ne sont pas en situation d'apprentissage dans un cadre institutionnel. Les transcriptions ont été opérées par l'équipe LIDILE ${ }^{5}$ (université Rennes 2) avec le logiciel CLAN, et pour l'instant une seule vérification a été effectuée. Le corpus ${ }^{6}$, déjà exploitable et destiné à être diffusé à la communauté scientifique, est en cours de finalisation. La base CIL est alimentée par les étudiants du Master 1 Linguistique et didactique des langues, ALE et FLE de l'université Rennes 2, dans le cadre de leur formation à l'analyse de l'interlangue.

\subsection{Méthode d'analyse}

23 Après une première écoute des 16 heures d'enregistrement, les passages sélectionnés ont été à nouveau écoutés puis analysés au moyen du logiciel Praat ${ }^{7}$ à partir des fichiers .wav. L'objectif était de vérifier si les allongements des syllabes et pauses de segmentation 
repérés à l'oreille étaient effectifs.

Les exemples sont référencés de la même façon que dans la transcription du corpus sous CLAN (le protocole de transcription se trouve en annexe) :

zho_yi_wa_93_f_13: zho $=$ sinophone $; \mathrm{yi}=$ premières lettres du prénom $; \mathrm{wa}=$ premières lettres du nom; 93 = année de naissance de l'interviewé-e; f pour femme (h pour homme) ; 13 pour l'année de l'enregistrement (2013). « ara » indique que le locuteur est arabophone, « eng » anglophone et « spa » hispanophone.

« ENQ » correspond à « enquêteur-trice » et « FLE » à « interviewé-e non natif-ve ».

La consigne donnée aux enquêteurs étant d'interviewer des locuteurs non natifs de niveau B2- $\mathrm{C} 1$, le référencement ne comporte pas d'indication de niveau.

\section{Premiers résultats}

\subsection{Interlocutions : moments où se signalent les différences prosodiques}

L'objectif des enquêteurs est de donner à entendre la parole des interviewés et ces derniers bénéficient donc dans les entretiens du temps de parole le plus important. Deux moments d'interlocution cependant donnent lieu à des échanges de longueur équivalente : les salutations initiales et les reformulations de question. Ces interlocutions signalent des différences prosodiques entre enquêteurs et interviewés.

\subsubsection{Salutations initiales}

Les salutations («Bonjour» ou «Bonjour X») mettent immédiatement en évidence des différences de prosodie entre natifs et non natifs: tout d'abord une absence d'allongement ou un allongement moindre de la dernière syllabe pleine de l'énoncé. Comme nous l'avons mentionné plus haut, la syllabe accentuée est plus longue que les autres syllabes : c'est ce que l'on vérifie dans le « bonjour » de l'enquêtrice mais pas chez l'interviewée.

\section{Exemple 1 :}

zho_yi_wa_93_f_13

*ENQ : bonjour.

*FLE : bonjour.

Figure 1. « bonjour » de l'enquêtrice

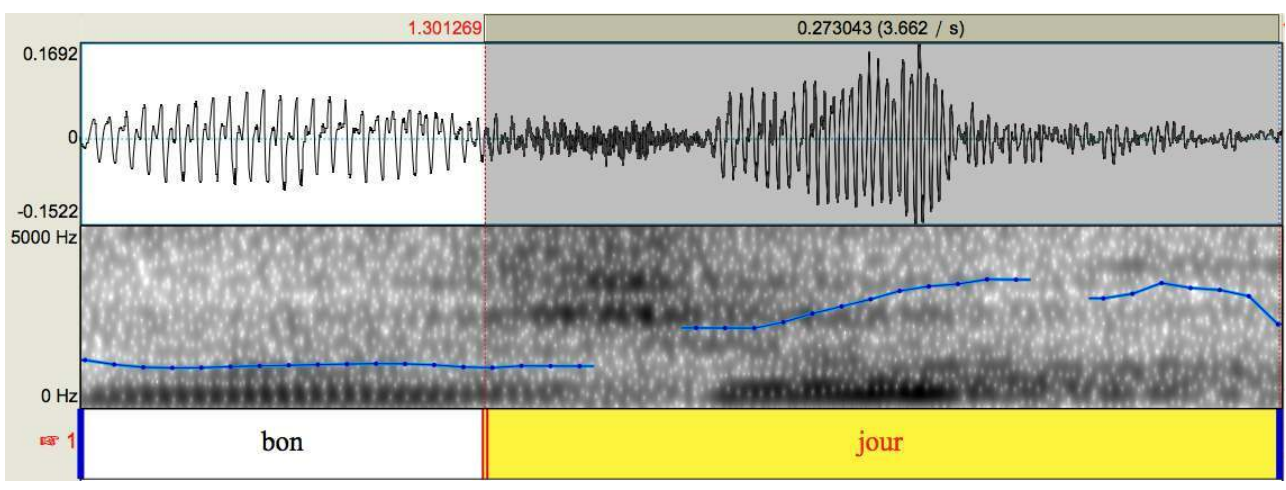

TIPA. Travaux interdisciplinaires sur la parole et le langage, 30 | 2014 
Figure 2. « bonjour » de l'interviewée zho_yi_wa_93_f_13

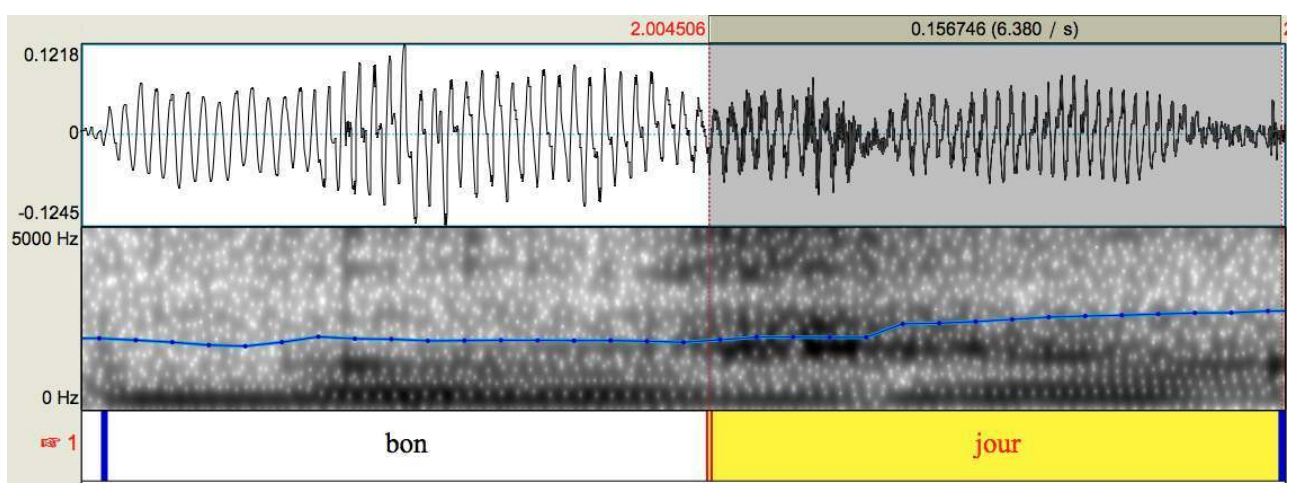

Dans ce premier exemple, la première syllabe ([โّ]]) de l'enquêtrice dure 138 millisecondes et la deuxième et dernière ([3uR]) $273 \mathrm{~ms}$. Tandis que les deux syllabes de l'interviewée sont respectivement de 165 et 156 ms, c'est-à-dire que sa deuxième syllabe est plus courte que la première. L'exemple 2 vient confirmer cette différence : la seconde syllabe de l'enquêtrice dure 587 et la première $136 \mathrm{~ms}$, elle est donc plus de quatre fois plus longue. L'interviewée quant à elle diminue sa seconde syllabe de $3 \mathrm{~ms}$ par rapport à la première, en passant de 258 à $255 \mathrm{~ms}$. Ces mesures confirment ce que perçoit l'oreille : ces deux enquêtrices allongent bien la dernière syllabe de «bonjour » ce qui n'est pas le cas de leurs interviewées.

\section{Exemple 2 :}

spa_ar_ca_73_f_10

*ENQ : bonjour.

*FLE : bonjour.

Figure 3. « bonjour » de l'enquêtrice

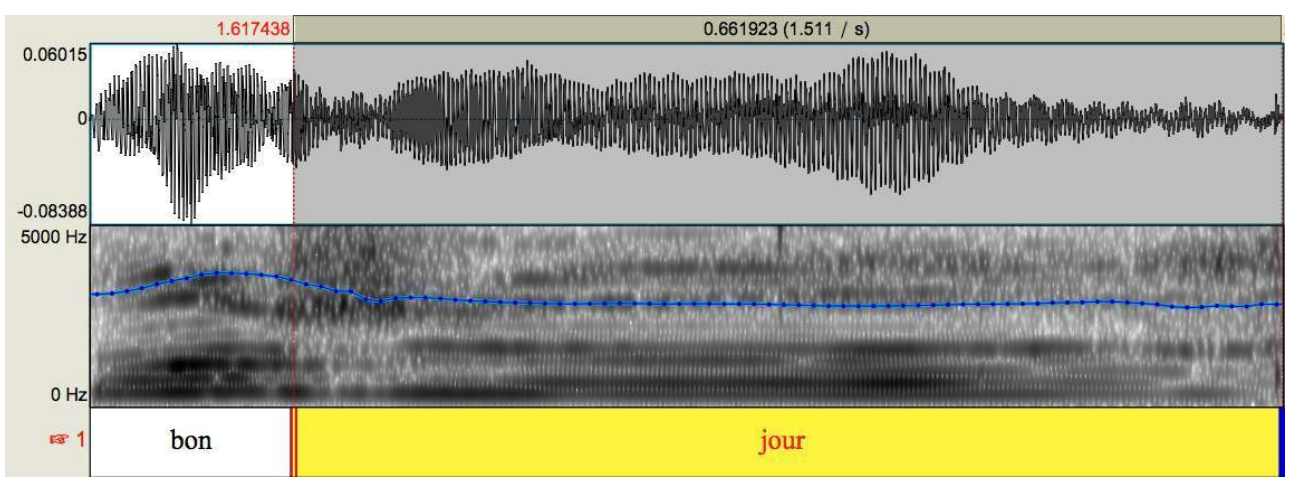


Figure 4. « bonjour » de l'interviewée spa_ar_ca_73_f_10

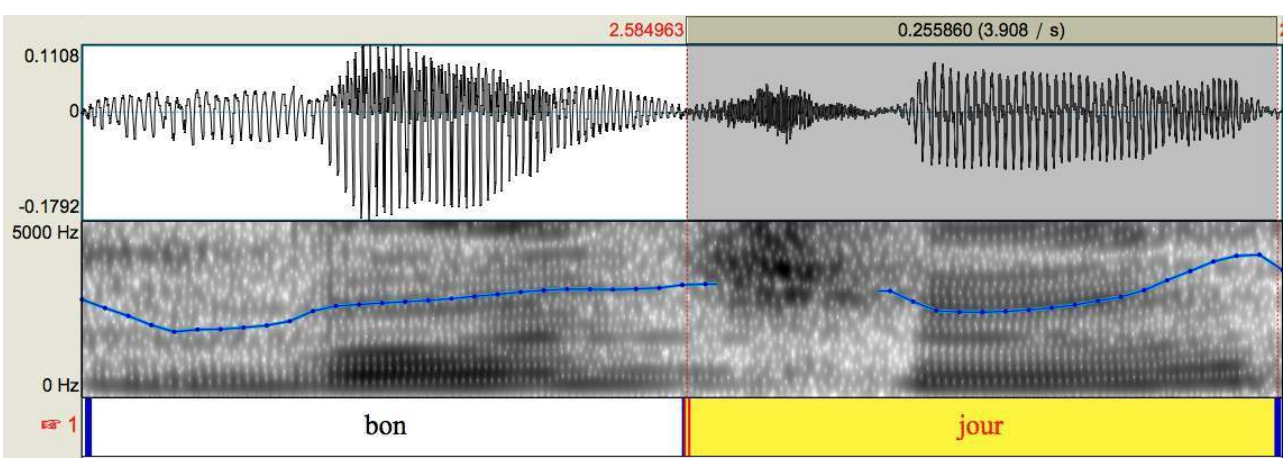

Les deux exemples qui suivent montrent une différence de traitement de la structure " Bonjour X », chez les natifs et les non natifs. Ces derniers font apparaître entre les deux éléments de l'énoncé, une pause de segmentation qui n'est pas produite par leur interlocuteur-trice.

\section{Exemple 1:}

spa_iv_ga_87_f_13

*ENQ : bonjour Yvonne.

*FLE : bonjour \# [150 ms] Thomas.

Figure 5. « bonjour Yvonne » de l'enquêteur

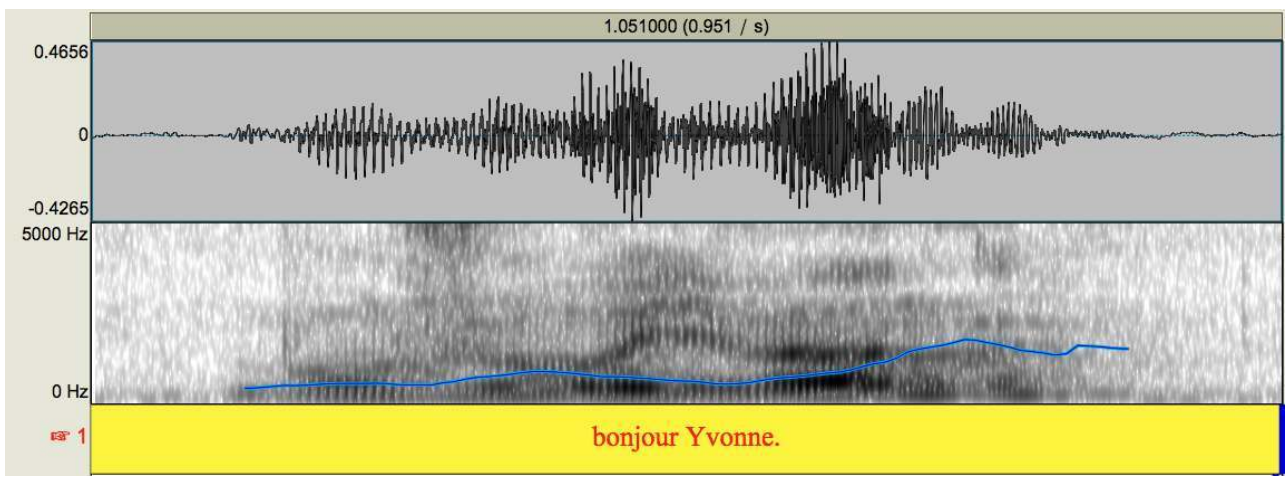

Figure 6 : « bonjour Thomas » de l'interviewée spa_iv_ga_87_f_13

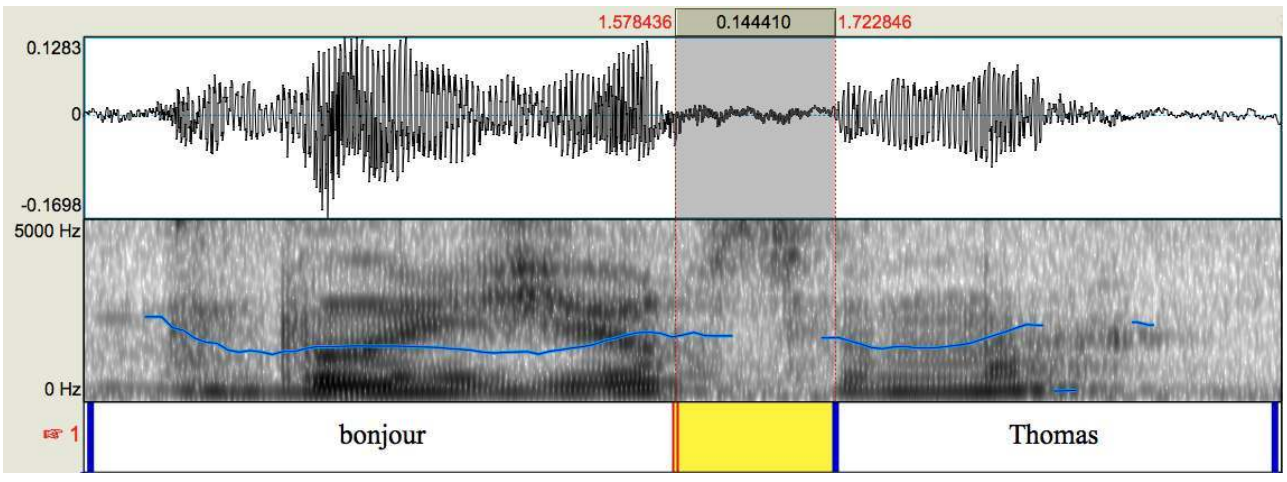

Dans l'exemple 2 aussi apparaît une pause entre «bonjour» et le prénom de l'interlocutrice, ici « Aurore ». 
zho_we_ch_88_f_10

*ENQ : bonjour Wenjie.

*FLE : bonjour \# [914 ms] Aurore.

\section{Figure 7. « bonjour Wenjie »}

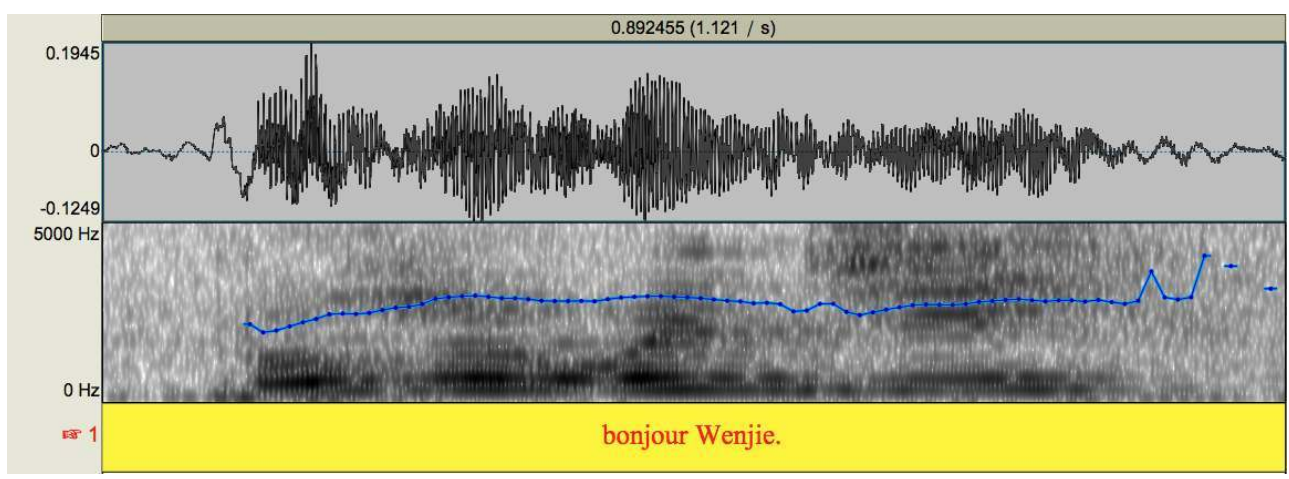

Figure 8. « bonjour Aurore » de l'interviewée zho_we_ch_88_f_10

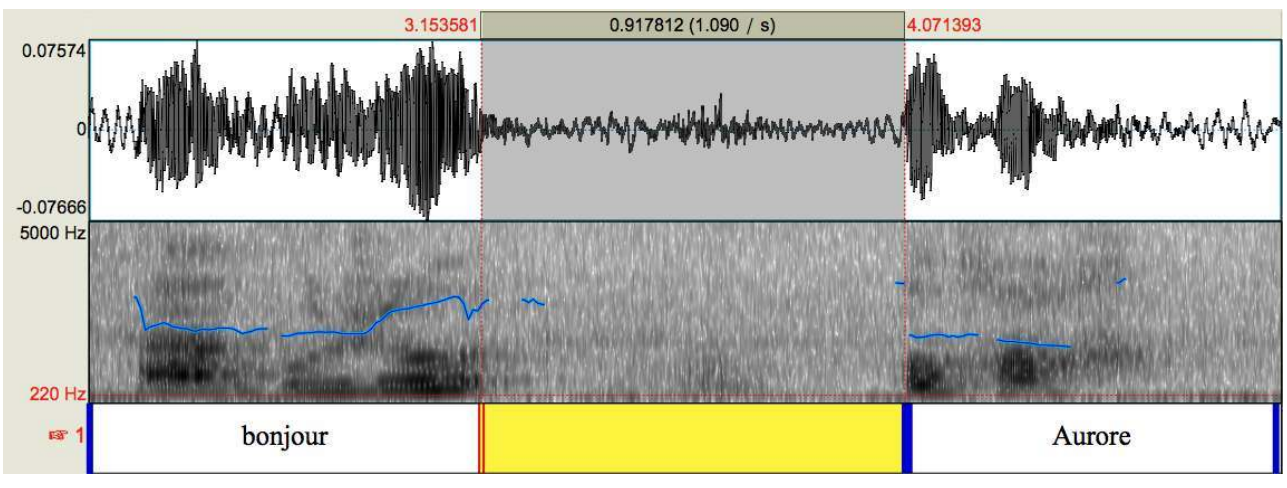

La pause, extraordinairement marquée dans l'exemple "bonjour Aurore ", peut en partie peut-être s'expliquer par le stress dû à la situation de communication et/ou à la difficulté liée à la prononciation du prénom de l'enquêtrice. Les deux exemples sont cependant représentatifs du fait que la chaîne parlée des locuteurs non natifs est beaucoup plus segmentée que celle des natifs, du fait aussi que souvent la pause ne marque pas une frontière de groupe, mais isole des mots. Un locuteur natif peut certes produire une telle pause mais le corpus montre que $75 \%$ des interviewés segmentent l'énoncé «bonjour X » contre à peine $25 \%$ des enquêteurs.

\subsubsection{Reformulations de questions}

Quand une question n'a pas été comprise et qu'elle est répétée par l'enquêteur-trice, la nouvelle formulation met en évidence une modification de la segmentation, et tout d'abord un allongement de la pause :

eng_ca_re_83_f_11

*ENQ : est+ce+que \# vous aimez beaucoup \# [0,80 ms] l'Angleterre ? 
${ }^{*}$ FLE : pardon.

*ENQ : est+ce+que \# vous aimez \# [226 ms] l'Angleterre?

D'autre part, que la reformulation réduise la question à ses éléments essentiels ou qu'elle l'allonge d'éléments explicatifs, la segmentation est augmentée.

spa_ga_ll_87_f_11

*ENQ : et tu as eu beaucoup d'heures \# de français dans la semaine?

*FLE : xxx pardon [x 2] xxx.

*ENQ : combien d'heures \# de cours \# de français \# tu avais dans la semaine?

zho_zh_bo_84_f_11

*ENQ: et hum \# en quoi est+ce+que \# Rennes \# c'est différent de là où d'où tu viens.

*FLE: euh encore.

*ENQ: en quoi \# est+ce+que \# cette ville là \# Rennes \# elle est différente \# de là

d'où tu viens \# de la Chine.

Dans le premier exemple, la question initiale comporte une pause tous les 2,5 mots et la reformulation, une pause tous les 2 mots. Dans le deuxième exemple, la segmentation est multipliée par trois, et dans le troisième, la pause intervient tous les 4,66 mots, puis tous les 3 mots dans la reformulation.

37 A cela s'ajoute, et tout particulièrement dans l'exemple suivant, un allongement marqué de la dernière syllabe pleine de groupe, allongement qui touche ici «faire », «bien», « sortir » et « musique».

spa_en_ro_92_h_13

*ENQ: hum hum d'accord \# et qu'est+ce+que tu fais \# pendant tes loisirs ici \# si tu fais pas de sport \# tu fais peut+être autre chose?

*FLE: en+fait j' ai pas compris \# qu'est+ce+que ça veut dire.

*ENQ: hum pendant ton temps libre \# est+ce+que tu as \# quelque+chose à faire \# est+ce+que tu aimes bien \# sortir \# écouter de la musique?

\subsubsection{Natifs et non natifs : un usage différent de la pause de segmentation}

Les passages de reformulation mettent donc en évidence que, pour faciliter la compréhension de son énoncé, un locuteur expérimenté (natif ou non) augmente le nombre et/ou la durée des pauses. Pauses qui constituent cette ponctuation « audible » [qui] permet à l'auditeur de progressivement orienter son attention à la fois vers la forme et le contenu du discours oral. (Faraco \& Kida, $2003: 169$ )

Les moments de salutations quant à eux, montrent que les non natifs recourent peu ou pas à l'allongement de la syllabe censée être accentuée et adoptent une segmentation qui fait du mot graphique l'unité prosodique de base, et ce au détriment du groupe accentuel. 


\subsection{Segmentation et morphosyntaxe}

\subsubsection{Une segmentation massive mais très rarement erronée}

ara_ca_ma_80_f_09

j'ai commencé \# à faire des cours \# euh de formation d' informatique \# Windows

Word \# Internet.

Le découpage prédit Windows \# Word \# Internet, est en fait réalisé : Windows Word \# Internet. Ceci fait passer l'énumération de trois à deux termes : le premier étant "Windows Word » et le second «Internet». La pause de segmentation attendue entre "Windows» et «Word» est comblée par un allongement $\mathrm{du}[\mathrm{z}]$ final de "Windows ». Celle qui sépare «Word » et «Internet », bien qu'elle soit minime $(0,40 \mathrm{~ms})$ est cependant perçue, mais uniquement parce qu'elle est attendue (Astésano, 2001).

1 En réalité, ce n'est pas l'absence mais la présence massive de la segmentation qui caractérise le corpus. Elle apparaît bien entendu à tous les moments d'hésitation liés à la recherche de la forme considérée comme juste. L'exemple suivant concerne le choix de la forme verbale :

spa_se_ma_93_h_12

on \# je n'ai pas de voiture \# j' ai \# je vais \# j' ai \# je va \# je vais \# en

bus et \# les soirs \# il+n'y+a+pas \# des bus \# je dois aller <en vélo> [x 2].

\subsubsection{Segmentation et morphologie : le cas du déterminant nominal}

Le choix du déterminant nominal est source de difficultés chez les locuteurs non natifs même s'ils sont de niveau avancé. Et de fait le corpus présente de multiples exemples d'hésitation et d'autocorrection concernant le choix de ce déterminant :

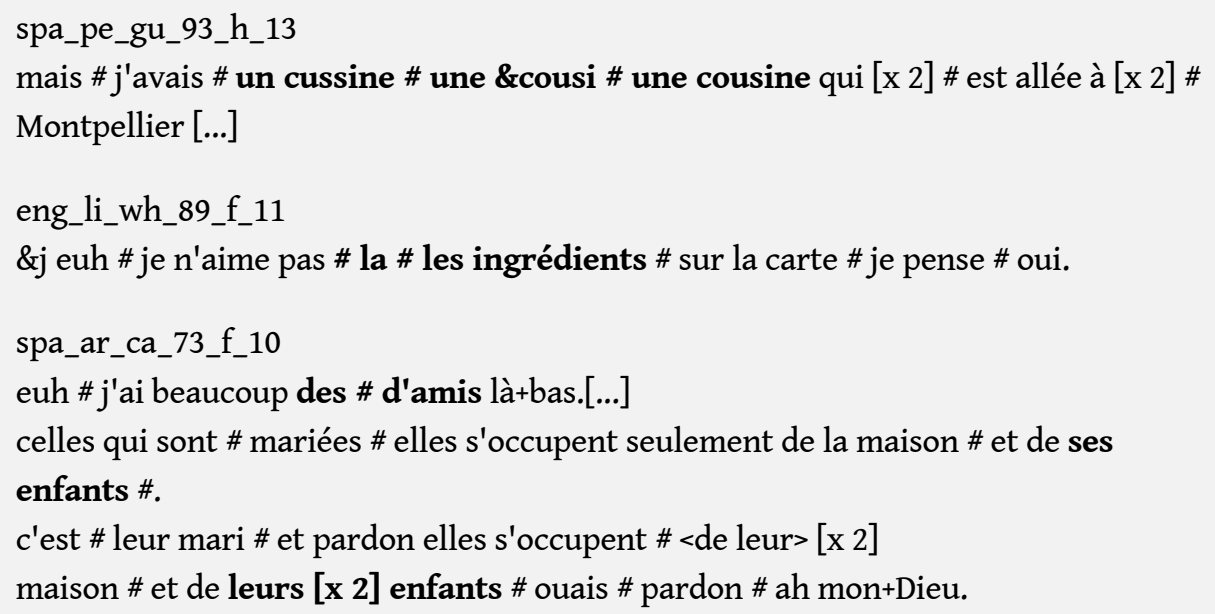


Mais c'est surtout l'absence du déterminant qui attire l'attention. Il s'agit tout d'abord des cas, dans l'interlocution, où un mot est repris, extrait de la question ou du commentaire de l'enquêteur-trice par l'interviewé-e :

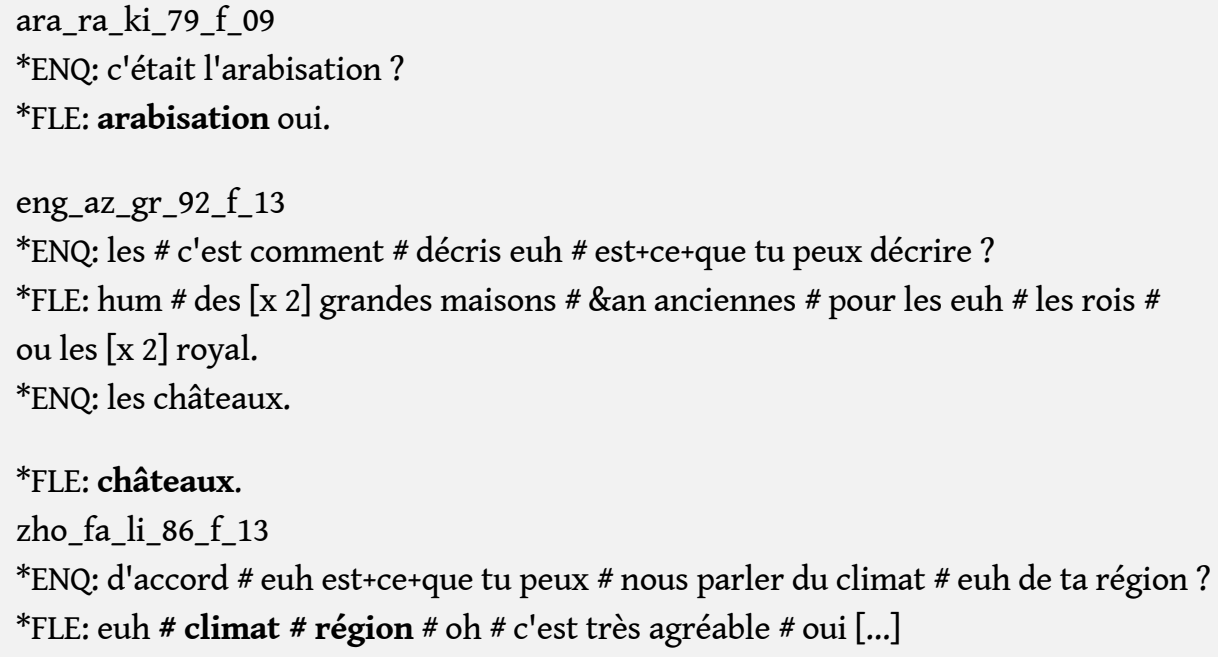

Ce qui a été compris, retenu et restitué ici, ce sont les mots " arabisation ", « châteaux " et « climat/région ». Les déterminants, présents dans l'énoncé de l'interlocuteur-trice, et qui pourraient être repris à l'identique (du moins dans les deux premiers exemples) ne le sont pas.

Dans l'extrait suivant et malgré le feed-back de l'enquêtrice, l'interviewée ne fait jamais précéder le nom d'un déterminant :

zho_zh_bo_84_f_11

*ENQ: tu aimerais bien \# avoir quoi.

*FLE: euh \# chaton \# euh \# chien.

*ENQ: un chaton et un chien [...]

*ENQ: et euh \# est+ce+que tu as \# un animal préféré.

*FLE: oui \# je préfère \# euh \# panda.

*ENQ: les pandas.

*FLE: oui \# c'est très \# c'est trop mignon.

*ENQ: est+ce+que tu pratiques un sport. [...]

*FLE: en Chine euh \# je [x 2] \# préfère \# euh \# natation \# et \# euh \# ping+pong \# oui et

[x 2] \# c'est tout et \# \&yo yoga \# aussi.

En revanche, le nom est chaque fois précédé d'un «euh» et d'une pause qui viennent comme remplacer ce déterminant. C'est aussi ce que montrent les exemples suivants dans lesquels la moitié ou les deux tiers des unités nominales sont complètes, dans lesquels aussi les absences de déterminants sont marquées prosodiquement par des silences de durées diverses :

zho_yi_wa_93_f_13

[...] les Français \# aiment des choses comme \# [0,29 ms] fromage \# [892 ms] beurre. 
eng_al_jo_86_f_10

il n'a pas \# une [x 2] \# route pour euh \# [176 ms] créativité d'ancrage, en perception et en expression. Dans les exemples d'interlocution, ces noms, qui sont des mots graphiques ici phonétiquement invariables, sont repris seuls, sans déterminant: «arabisation », « château(x)» (peut-être la transcription pourrait-elle s'abstenir $d u$ « $x$ » de " château »), « climat », " région ». Cela confère à ces noms une valeur autonymique - non voulue - et les réponses pourraient être paraphrasées ainsi : " "arabisation", oui, est le mot juste»; " "château(x)" est le mot que je cherchais ; " "climat" et "région" sont les mots que j’ai compris et les réalités dont il m’est demandé de parler ».

Dans les exemples où « fromage », « beurre ", « créativité » sont utilisés sans déterminant c'est aussi à la base lexicale qu'est donnée la primauté, au détriment du déterminant et donc de l'unité nominale. La prosodie porte cependant la marque de cette absence : la pause et/ou le « euh » signalent le caractère incomplet de l'unité et la conscience qu'en a vraisemblablement le-la locuteur-trice.

\subsubsection{Segmentation et syntaxe : le cas du « que » conjonction de subordination}

L'oral est le lieu de la parataxe et la juxtaposition y prévaut : le corpus dont il est ici question le montre bien. Les mots subordonnants, peu fréquents, sont essentiellement " quand » et « parce que », et en ce qui concerne les relatifs, seuls « qui » et « que » sont réellement présents (et avec des confusions récurrentes). Par ailleurs, l'utilisation du « que » de subordination est très instable. Les verbes du type « je crois », « je pense », « je trouve » ou "j'espère » (induits par un entretien dont les questions portent sur la perception des différences interculturelles, le parcours et les projets des interviewés) sont fréquents, mais souvent non suivis du « que » subordonnant.

eng_la_sk_86_f_10

je crois maintenant \# je vais rentre \# euh \# aux États_Unis.

zho_ju_zh_87_h_12 audio

$x x x$ je crois $i l+n^{\prime} y+a+$ pas de loisirs \# car il $+n^{\prime} y+a+$ pas \# de piano \# il+n' $y+a+$ pas de ping +pong $\mathrm{xxx}[\ldots]$

eng_ca_re_83_f_11

j' espère je peux travaille \# comme une professeur \# de anglais.

zho_yi_wa_93_f_13

en France \# je trouve les Français \# aiment des choses comme \# fromage \# beurre.

Cependant, signe d'un apprentissage en cours, à quelques secondes (à quelques mots) d'intervalle peuvent se succéder formes incomplète et attendue :

eng_al_jo_86_f_10

je pense des élèves \# les élèves \# pensent que je suis \# un+peu fou \# folle. 
ara_ra_ki_79_f_09

moi \# je [x 3] \# pense que \# euh c'était \# une \# bon \# chose \# mais \# il était \# il

[x 2] est dépassé \# la limite \# c'est [ $\mathrm{x}$ 2] bon \# de faire l'arabisation \#

mais en conservant \# aussi euh \# une bon étude de \# des langues étrangers \#

mais [x 2] \# moi je crois oui. [...]

euh \# on est \# producteurs de pétrole \# mais on n'a pas beaucoup de pétrole \#

en Syrie \# je crois pas que \# euh \# je crois \# on [x 2] \# produit euh \#

à+peu+près \# quatre cent \# mille \# barils \# chaque jour \# et ça c'est peu [...]

Par ailleurs, il faut noter qu'un « je pense » non introductif et ne demandant donc pas de subordonnant, peut à juste titre être inséré dans le cours d'un énoncé comme dans :

eng_li_wh_89_f_11

\&j euh \# je n'aime pas \# la \# les ingrédients \# sur la carte \# je pense \# oui.

Mais les deux formes - ici des «je crois» introductifs demandant un «que» subordonnant, et des «je crois", en fin d'énoncé ou en incise - entrent aussi en concurrence et donnent des énoncés partiellement corrects (et partiellement erronés) du type :

ara_di_ma_83_f_09

*ENQ : pour en revenir à la Syrie \# est+ce+que tu peux nous parler des euh

\# de sa situation économique \# quelles sont les ressources \# syriennes \#

c'est le tourisme principalement ? le tourisme \# l'agriculture.

${ }^{*} \mathrm{FLE}$ : euh oui <je crois> [x 2] \# l' agriculture \# n'est+ce+pas ?

*ENQ : quelle sorte d' agriculture \# il+y+a en Syrie?

*FLE : et il+y+en+a \# de \# maïs \# je crois.

zho_yi_wa_93_f_13 audio

je crois c'est intéressant \# et les questions \# je crois \# c'est beaucoup plus grand \# que ce+que j' ai prévu.

51 Enregistrements et transcriptions mettent en évidence, d'une part, que l'incise de type « je crois », insérée en milieu ou en fin d'énoncé, est toujours utilisée de façon attendue, et d'autre part que la segmentation ponctue pertinemment ce syntagme au moyen de deux pauses. Parallèlement, lorsque le "je crois » est utilisé sans le subordonnant attendu, il n'est pratiquement jamais suivi d'une pause : l'absence du « que » n'est en effet quasiment jamais marquée prosodiquement par une segmentation pouvant correspondre à une hésitation ou être interprétée comme la volonté de mettre en valeur la suite de l'énoncé. Il y a donc bien ici corrélation entre syntaxe et prosodie : toutes deux attendues elles se renforcent, mais lorsque la syntaxe est erronée, la segmentation, inexistante, ne compense pas l'absence du subordonnant. Non identifiée comme nécessaire, la subordination n'est pas actualisée dans le discours. Il est probable que l'énoncé: «je pense que je vais rentrer aux Etats-Unis » attendu (réalisé « je pense je vais rentrer ») est en concurrence dans l'interlangue du locuteur, avec « je pense rentrer aux Etats-Unis »; tout comme l'énoncé « je trouve que les Français aiment des choses » (réalisé «je trouve les Français aiment des choses ») est en concurrence avec un énoncé du type : «je trouve les goûts des Français bizarres /je trouve bizarres les goûts des Français ». On peut bien 
sûr aussi penser à un calque de l'anglais ("I think I'm going to go back » ou "I find French people like things ») mais il est troublant de voir que cette erreur, présente dans les énoncés des anglophones, l'est aussi chez les sinophones et les arabophones du corpus, locuteurs pour lesquels cette interférence avec l'anglais paraît difficile à établir.

\subsection{Allongements et segmentations prédits réalisés}

Si l'écoute du corpus permet de mettre en évidence des échecs, elle permet aussi bien sûr l'observation de réussites.

\subsubsection{Dans la restitution de discours de natifs}

Deux des enregistrements du corpus fournissent des passages de restitution de discours de natifs. Dans l'un des deux, l'interviewée, assistante de langue dans un lycée, explique vouloir éviter les exercices, selon elle ennuyeux et sans intérêt, qui consistent pour les apprenants à décrire leur week-end, type d'exercice qu'elle a pratiqué en tant qu'apprenante :

eng_al_jo_86_f_10

quand \# j'ai pris \# français \# \& j j' ai appris français.

j'ai détesté les [ $\left[\begin{array}{l}2 \\ 2\end{array}\right.$ \# petites histoires \# et les petits exercices

qui \# étaient toujours le même \# qu'est+ce+que tu fais \# ce weekend ?

La prosodie de l'énoncé de l'exercice, à savoir « qu'est-ce que tu fais \# ce week-end? », restitution d'une consigne prononcée pendant un cours de français, diffère de ce qui précède et de ce qui suit en ce qu'elle est très typiquement française. L'interrogation est ostensiblement segmentée en deux groupes accentuels «qu'est-ce que tu fais » et «ce week-end » dont les dernières syllabes [f $[\varepsilon]$ et $[k \varepsilon n d]$ sont très allongées.

Un peu plus loin dans l'entretien, interrogée sur le système éducatif français, cette locutrice répond:

en France \# c'est toujours \# ok.

tu [x 2] \# écris une paragraphe de \# dix lignes.

pas neuf \# et pas onze \# mais dix lignes.

et <il s' agit> [x 2] quoi \#

les films \# le cinéma \# qu'est+ce+que tu fais le weekend \# c'est toujours ça.

A la fin de la réponse, la prosodie signale le «c'est toujours comme ça " comme un commentaire personnel et reproduit (mime) celle de l'enseignant-e dans «les films \# le cinéma \# qu'est+ce+que tu fais le weekend \# ». La locutrice non seulement respecte les groupes accentuels et l'allongement des syllabes, mais elle tend vers l'isochronie : pour que la longueur, sept syllabes, du dernier groupe accentuel («qu'est-ce que tu fais le week-end ») se rapproche de celles de chacun des deux premiers groupes, respectivement de deux et quatre syllabes, elle accélère sa vitesse d'élocution.

L'autre entretien marqué par cette modification de la prosodie, restitue un dialogue de l'interviewée avec un francophone natif. A la question de l'enquêtrice lui demandant si elle a eu l'occasion de parler français avec des Français au Mexique, elle répond par le récit de sa rencontre avec deux Français dans un bus à Guadalajara. 
spa_ar_ca_73_f_10

et \# j'observais \# que \# deux garçons \# ont monté aussi \# l'autobus \# et \# pour

le \# tu sais \# pour le \# les \# visages \# qui sont un+peu différents.

et je me demandais s' ils @ét \# étaient \# mexicains ou pas \# mais bon \#

heureusement \# ils ont commencé à parler.

et j'écoutais le français \# et alors je disais \# ah j'adore parler avec eux.

et \# la \# le \# la situation a été un+peu bizarre \# parce+que un de ces

garçons \# et xxx s'avait \# assis \# près+de moi.

et il m'avait \# frappé une jambe \# euh \# accidentellement \# et

il m'avait dit \# pardon \#.

et $\mathrm{j}$ 'avais dit \# pas de soucis \# [...]

Dans la restitution de l'échange « il m'avait dit \# pardon \# et j'avais dit \# pas de soucis \# », les pauses qui suivent les verbes «dire" sont très marquées. D'autre part, les syllabes accentuées de "pardon» et de "pas de soucis » font respectivement 235 et $220 \mathrm{~ms}$ alors que les syllabes qui les précèdent sont de $140 \mathrm{~ms}$ pour la première syllabe de " pardon » et de 110 et $0,60 \mathrm{~ms}$ pour les deux premières syllabes du groupe «pas de soucis ». On peut noter que le «pas de soucis » prononcé [padsusi] opte pour la chute du [ə] du français non méridional renforçant l'impression de cohésion du groupe accentuel. Restituer le discours du locuteur natif tout comme restituer son propre discours dans l'interlocution avec un natif conduisent donc la locutrice à adopter le découpage et l'allongement attendus.

\subsubsection{Dans les énumérations, en particulier de toponymes français}

S'exprimant sur leurs voyages, les interviewés énumèrent des noms de lieux, en France et à l'étranger, en particulier des monuments, des villes et des régions :

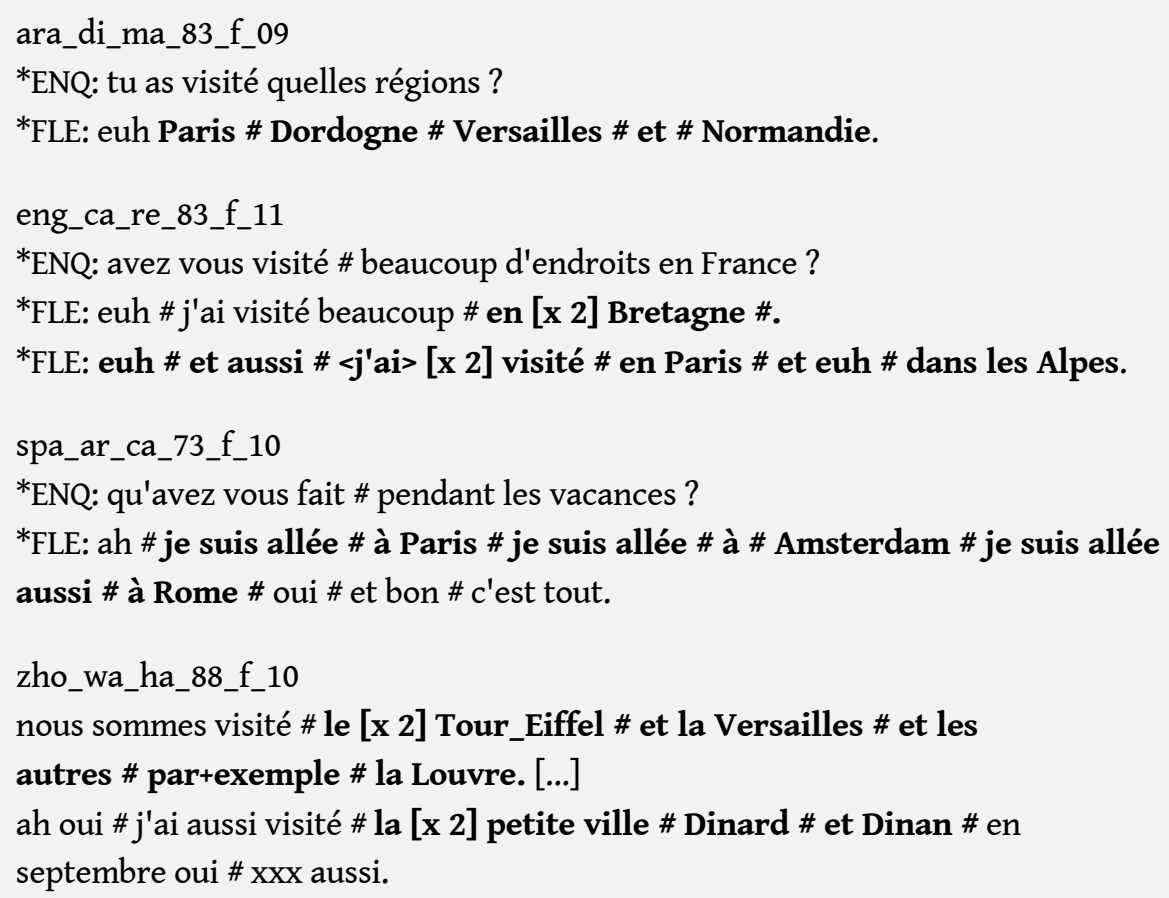


60

Lemples ci-dessus mettent en évidence les difficultés liées au choix de l'article et de la préposition devant les noms de lieux, mais montrent surtout que les énumérations de toponymes sont ponctuées par la segmentation et l'accentuation attendues.

D’une part, lorsque le nom de lieu est précédé d'une préposition et/ou d'un article, à part dans l'exemple « à \# Amsterdam », aucune pause ne sépare jamais le toponyme de ce qui le précède (par exemple, « en Bretagne », « à Paris », « dans les Alpes » ou « la Louvre »), d'autre part les toponymes désignant des villes ou régions françaises sont marqués par l'allongement de leur dernière syllabe pleine: qu'il s'agisse de "Paris », "Dordogne », « Dinan », ou « Versailles » par exemple, la remarque valant aussi pour « le Tour Eiffel». Seul d'ailleurs le toponyme « les Alpes » est prononcé de façon erronée : [lદzalps].

Et dans l'énoncé : « je suis allée \# à Paris \# je suis allée \# à Amsterdam \# je suis allée aussi \# à Rome ", les dernières syllabes des groupes "à Paris ", "à Amsterdam " et "à Rome " durent 485, 136 et 321 ms. La syllabe finale de «Paris » est de très loin la plus longue ; «Amsterdam », dont la prononciation n'est pas francisée, n'est pas caractérisée par l'allongement de [dam], alors que le choix de «Rome » et non de «Roma » conduit à l'allongement de la syllabe finale.

L'allongement concerne donc la prononciation des toponymes français ou francisés : les interviewés, séjournant ou ayant séjourné en France, sont imprégnés de la prononciation de ces noms propres fréquemment entendus et prononcés. Mais la seule exposition à la langue est rarement suffisante : le respect de l'accentuation est donc aussi lié au fait que ces noms, non susceptibles d'être fléchis, et obligatoirement déterminés, quand ils le sont, par un article défini (dont seul le genre peut poser question), ont une forme stable, ce qui allège cognitivement la réflexion du locuteur. Enfin, la forme même de l'énumération, qui fait se succéder de façon identique des unités de même fonction, permet au locuteur d'adopter et de répéter un même modèle prosodique.

\subsubsection{Dans la réitération des structures}

61 Ce qui est vrai pour les énumérations de toponymes se vérifie dans des énumérations d'autres types d'unités, nominales ou verbales. Dans les exemples suivants ont été mesurées d'une part la durée de la syllabe finale de groupe (qui apparaît dans l'exemple, entre crochets) et d'autre part la durée de la syllabe (quand le groupe ne comporte que deux syllabes) ou des deux syllabes précédentes. Les durées moyennes des syllabes non accentuées et des syllabes accentuées sont indiquées à la suite de chaque exemple.

ara_mo_sa_79_f_09

[...] avec les participations \# avec ma famille [430 ms] \# mes frères [298 ms] \# ma mes sœurs $[625 \mathrm{~ms}]$ \# mon père $[165 \mathrm{~ms}]$ \# et ma mère [345 ms] \# c'est ça \# je trouve que mon temps \# c'est pleine \# toujours.

Durée moyenne des syllabes non accentuées : $157 \mathrm{~ms}$

Durée moyenne des syllabes accentuées : $372 \mathrm{~ms}$

ara_sa_ka_80_f_09

il y avait [...] une plage de pierre $[384 \mathrm{~ms}]$ \# et de sable $[1100 \mathrm{~ms}]$ \# euh bon \# c'était vraiment \# très \#

joli \# et les forêts [247 ms] \# les montagnes [417 ms] \# les paysages [390 ms] \# étaient très \# formidables. 
Durée moyenne des syllabes non accentuées : $128 \mathrm{~ms}$

Durée moyenne des syllabes accentuées : $507 \mathrm{~ms}$

spa_ar_ca_73_f_10

le français \# je l'aimais bien \# ah \# parce+que \# on disait \# ah c'est romantique [297

$\mathrm{ms}]$ \#

c'est musical [460 ms] \# [...] et \# c'est la langue de l'amour [616 ms] \# des choses

comme+ça \# n'est+ce+pas.

Durée moyenne des syllabes non accentuées : $151 \mathrm{~ms}$

Durée moyenne des syllabes accentuées : $457 \mathrm{~ms}$

spa_na_kl_87_f_12

j'aime bien \# aller au cinéma [ $549 \mathrm{~ms}$ ] \# euh j'aime bien \# sortir avec des amis [713 ms] \#

euh j'aime bien lire [559 ms] \# beaucoup \#.

Durée moyenne des syllabes non accentuées : $108 \mathrm{~ms}$

Durée moyenne des syllabes accentuées : $607 \mathrm{~ms}$

zho_yi_wa_93_f_13

et avec elle \# <j' ai> [x 2] appris beaucoup \#.

comment elle fait [518 ms] \# comment elle parle [365 ms] \# avec les gens A

A2014-12-12T15:39:00_[383 ms] \#.

Durée moyenne des syllabes non accentuées : $197 \mathrm{~ms}$

Durée moyenne des syllabes accentuées : $422 \mathrm{~ms}$

Les exemples montrent que la syllabe accentuée est en moyenne de 2 à 5,6 fois plus longue que les syllabes inaccentuées qui la précèdent.

Si l'on extrait les groupes accentuels marqués par l'allongement de la dernière syllabe, il apparaît qu'il s'agit de groupes, juxtaposés ou coordonnés, construits à l'identique :

ma famille / mes frères / ma mes søurs / mon père /et ma mère /

une plage / de pierre / et de sable /

les forêts / les montagnes / les paysages /

c'est romantique /c'est musical /c'est la langue de l'amour/

j'aime bien aller au cinéma / j'aime bien sortir avec des amis / j'aime bien lire /

comment elle fait / comment elle parle /avec les gens /

Deux des énoncés modifient légèrement la structure adoptée, comme si, une fois « rôdée " la forme pouvait être adaptée. La modification concerne donc, logiquement, le dernier groupe accentuel de l'énumération.

Dans « j'aime bien / aller au cinéma / sortir avec des amis / j'aime bien lire / ", l'ajout de «j'aime bien » au début du troisième terme de l'énumération, atténue le déséquilibre lié au choix du verbe monosyllabique «lire». Le rythme $(6+7+3)$ a été préféré - et est bien préférable - à $(6+7+1)$.

L'énumération « c'est romantique / c'est musical [...] / c'est la langue de l'amour / », $(4+4$ 
+6), quant à elle, respecte le principe de progression (Lacheret, 2011) selon lequel la taille des groupes n'est pas équivalente mais, au contraire, augmente progressivement au fur et à mesure de l'énoncé, progression qui, dans l'exemple ci-dessus, met en relation, comme le note Lacheret $(2011: 6)$, longueur des groupes et densité de l'information.

Très rares sont les énoncés qui maintiennent, alors qu'intervient un élément complètement différent, le rythme adopté dans la répétition. C'est cependant le cas de l'exemple où " comment elle fait / comment elle parle / " s'achève par " avec les gens ", mettant ainsi en place un rythme $(4+4+4)$ parfaitement équilibré.

En dehors du fait que certains sons consonantiques comme le [R] (Wioland, 1983), favorisent l'allongement final (et le corpus contient de nombreux entretiens dans lesquels seuls des mots comme «faire », « affaires » ou « sport » sont marqués par l'allongement), il apparaît ici que c'est bien la répétition d'une même structure qui favorise l'émergence de la prosodie attendue. ${ }^{8}$

Un autre phénomène confirme ce constat: lorsqu'un même élément est répété par le locuteur, l'allongement apparait, ou est augmenté, sur la finale du groupe accentuel, lors de la répétition.

spa_al_ga_79_h_12

des instruments \# chaque fois \# sont \# euh \# nouveaux [266 ms] \# sont nouveaux [334 $\mathrm{ms}]$.

spa_ar_ca_73_f_10

ouah \# le \# la nourriture \# est \# plus \# pimentée [134 ms] xxx pimentée [346 ms].

71 Dans ces exemples, au moyen de l'allongement sur "nouveaux » et "pimentée ", le locuteur arrête et confirme son choix (de mot et de prononciation) et rend compte de la valeur pragmatique de la prosodie.

La répétition, tout comme l'énumération, permettent donc au rythme de se faire jour. Lorsque les termes de l'énumération, parce que noms propres invariables, ne demandent pas à être fléchis, ou que la structure choisie ne demande qu'à être répliquée dans la répétition, l'actualisation des pauses et des allongements s'en trouve facilitée. Et lorsque la répétition vient confirmer un choix lexical et/ou morphologique, c'est «naturellement » au moyen de la prosodie (à moins que ce ne soit la morphologie qui vienne confirmer le rythme).

\section{Conclusion et pistes didactiques}

Les éléments présentés ici correspondent à de tout premiers constats. Ils mettent cependant en évidence que le corpus d'interlangue envisagé est particulièrement instructif en ce qu'il permet une analyse à la fois synchronique et diachronique. Les entretiens donnent en effet à entendre des réussites et des erreurs de locuteurs non natifs à un moment donné de leur apprentissage, mais aussi des performances relevant à la fois de leur niveau et d'un niveau antérieur. En témoignent des énoncés qui, à quelques secondes d'intervalle, actualisent ou non la structure attendue ou la prosodie prédite.

Concernant les proéminences de l'oral envisagées, allongement de la syllabe accentuée et pauses de segmentation, le corpus vient conforter les intuitions et constats empiriques des enseignants-tes de FLE. Il confirme le non allongement de la syllabe finale accentuée, 
et l'introduction de pauses de segmentation non prédites. Il fait apparaître que, pour le-la locuteur-trice non natif-ve, l'unité prosodique de base est souvent le mot graphique, et ce au détriment et de la syllabe et du groupe accentuel.

Cette prééminence du mot graphique est visible, entre autres, dans les passages de salutations et les moments d'interlocution où un mot -le mot entendu et compris - est repris seul, non précédé d'un déterminant. De façon générale, le fait que l'absence du déterminant, non systématique, soit souvent comme comblée par un «euh» ou une pause, signale un apprentissage en train de se faire, la non actualisation d'un format pourtant déjà là. En revanche, si l'absence du " que » subordonnant montre aussi un état de l'interlangue des locuteurs, cette erreur n'est pas signalée par la prosodie. Il y a confusion entre subordination et juxtaposition, et la prosodie ne vient pas au secours de la syntaxe.

76 Le corpus révèle aussi des réussites : elles concernent tout d'abord la restitution de discours de natifs, mais aussi l'énumération, la répétition d'une même structure ou la confirmation d'une formulation. Dans tous ces cas sont effectifs à la fois l'allongement de la syllabe finale et la pause de segmentation qui étaient attendus. Dans ces cas aussi apparait l'isochronisme et devient effectif un rythme qui respecte à la fois les principes d'eurythmie et de progression. Ces réussites apparaissent de façon inégale : une analyse systématique de toutes les formes de réitérations du corpus permettrait de mettre en évidence (la prise en compte de la L1 des locuteurs serait alors peut-être utile aussi) quelles structures font surgir la prosodie attendue.

Convergence - et l'idée, pour n'être pas nouvelle n'en est pas moins essentielle - du phonétique et du morphosyntaxique : tel devrait être le mot d'ordre de l'enseignementapprentissage du FLE. Cette convergence pourrait être mise en évidence par un travail d'écoute et de production, dès le début de l'apprentissage, de l'allongement de la syllabe finale d'un mot comme "bonjour "; ce travail devrait être immédiatement suivi de l'explication de la notion de groupe accentuel et de ce qu'elle implique pour le déplacement de l'accent dans le groupe «bonjour+prénom ». Il s'agirait ainsi de profiter de ce tout début d'apprentissage (et/ou de ce tout début de cours) pour expliciter les caractéristiques de l'accent en français, et faire en sorte que l'enseignementapprentissage des actes de langage soit associé à des moments de parole prosodiquement authentiques.

Car à la notion de crible phonologique (Troubetskoy, 1949) pour le segmental, correspond celle de "surdité accentuelle » pour le suprasegmental (Dupoux, 2002) : nous percevons ce que nous avons appris à percevoir et ce que nous avons l'habitude de percevoir. Et si pour Delattre (1966: 142), [l]e français donne l'impression d'une succession de syllabes sensiblement égales dont le rythme ininterrompu cache malicieusement les limites des mots, il apparaît que l'unité de base, pour un non natif est souvent le mot et non la syllabe. Ceci est visible en production dans le non respect de l'isosyllabicité et de l'allongement de la syllabe finale, méconnaissance qui entraîne la suppression ou quasi suppression de certaines syllabes (et qui fait par exemple que "furieux» est prononcé [fRjø], ou "comment dire», [kJmdi]). Il semble donc nécessaire de mettre en place un entraînement systématique à la syllabation par la scansion, ce qui permet par ailleurs un travail sur le segmental.

Il parait aussi indispensable de présenter la langue comme un système dont l'unité de base, en morphologie et en prosodie, n'est pas le mot graphique mais le groupe, composé pour l'unité nominale par exemple, au minimum d'un déterminant et d'un nom. Il est 
nécessaire également de montrer qu'incise et subordonnée par exemple, ne sont interchangeables ni en syntaxe ni en prosodie. Car le corpus confirme une différence importante entre non natifs et natifs : alors que chez les natifs la prosodie prend le relais d'une syntaxe approximative ou tout simplement « ordinaire », c'est rarement le cas chez les non natifs. Et il s'agit bien là d'une raison supplémentaire pour que l'enseignementapprentissage du FLE ne passe surtout pas sous silence l'intimité et la solidité des liens qui unissent ces paramètres linguistiques.

Or in :

\section{BIBLIOGRAPHIE}

Astesano C. (2001) Rythme et accentuation en français, L'Harmattan, Paris, 337 p.

Callamand M. (1973) l'Intonation expressive, exercices systématiques de perfectionnement, Hachette/

Larousse, collection Le Français Dans le Monde, 174 p.

Carton F. (1974) Introduction à la phonétique du français, Bordas, Paris, $250 \mathrm{p}$.

Dahan D. \& Bernard J.M. (1996) Interspeaker Variability in Emphatic Accent Production in French in Language and Speech, Vol. 40 (2), 141-201.

Delais-roussarie E. (2000) Vers une nouvelle approche de la structure prosodique, in Langue française, $\mathrm{n}^{\circ} 126$, p. 92-112, Armand Colin, Paris.

Delais-roussarie E., Yoo H. et Post B. (2011) Quand frontières prosodiques et frontières syntaxiques se rencontrent, in Langue française, $\mathrm{n}^{\circ}$ 170, p. 29-44, Armand Colin, Paris.

Delattre P. (1962) Some Factors of Vowel Duration and their Cross-Linguistic Validity in Journal of the Acoustic Society of America, Vol. 34, 1141-1143.

Delattre p. (1966) Studies in French and comparative phonetics, Mouton, La Haye, (Recueil de 32 articles sur la phonétique française, articulatoire et acoustique), $286 \mathrm{p}$.

Dupoux E., Peperkamp S. and Sebastian-galles N. (2002) A robust method to study stressdeafness, in Journal of the Acoustical Society of America, vol. 110, n³, Pt.1 : 1606-1618. DOI : $10.1121 / 1.1380437$

Faraco M. \& Kida T. (2003) Interaction entre prosodie didactique et prise de notes en L2, in Arob@se, [Arob@se, www.arobase.to, volume 1-2, p. 167-179].

Fonagy I. (1980) L'accent en français : accent probabilitaire, in L'accent en français contemporain (Studia Phonetica), Vol.15, I. Fónagy \& P. Léon (eds.), p. 123-233.

Gadet F. (1989) Le Français ordinaire, Armand Colin, Paris, 192 p.

Intravaia P. (2000) Formation des professeurs de langue en phonétique corrective. Le système verbo-tonal, CIPA (3ème tirage), Mons, $283 \mathrm{p}$

Lacheret A. (2001) La prosodie au cœur du verbal, in Rééducation Orthophonique, n² 246, p. 87-104. $<$ halshs-00636556> p. 1-14.

Lauret B. (2007) Enseigner la prononciation du français : questions et outils, Hachette FLE, Paris, 192 p. 
Leon M. \& Leon P. (2002, 1997 pour la première édition) La Prononciation du français, Nathan, Paris, $127 \mathrm{p}$.

Leon P. (2001, 1992 pour la première édition) Phonétisme et prononciations du français, Nathan, Paris, $192 \mathrm{p}$.

Malmerg B. (2002, 1954 pour la première édition) La Phonétique, Presses Universitaires de France, Paris, $127 \mathrm{p}$.

Martin P. (2008) Contraintes rythmiques et syntaxiques dans la relation prosodie-syntaxe, in Travaux linguistiques du CerLiCO, Grammaire et prosodie, $n^{\circ} 21$, p. 14-25, PUR, Rennes.

Pasdeloup V. (1990) Modèle de règles rythmiques du français appliqué à la synthèse de la parole, thèse de Doctorat, Université de Provence I, 386 p.

Seguinot A. (1976) L'accent d'insistance en français standard in Studia Phonetica, Vol. 12, p.1-58.

Troubetzkoy N. S. (1949) Principes de phonologie, trad. Cantineau J., Klincksieck, (réimpression. 1964), Paris, 396 p.

Vaissiere J. (2010) Le français, langue à frontières par excellence, in Frontières, du linguistique au sémiotique, ouvrage collectif initié par Nelly Andrieux-Reix, dirigé et édité par Dominique Delomier en collaboration avec Mary-Annick Morel, éditeurs Lambert Lucas, Limoges. [hal.inria.fr/docs/00/68/29/80/PDF/vaissiere_2010-frontieres.pdf]

Wenk B.J. \& Wioland F. (1982) Is French really syllable-timed? in Journal of phonetics, Vol 10, p. 193-216.

Wioland F. (1991) Prononcer les mots du français, Hachette, Paris, 127 p.

Wioland F. (1983) La Rythmique du français parlé, Publications de l'Institut international d'études françaises, $\mathrm{n}^{\circ} 7$, Strasbourg, $155 \mathrm{p}$.

\section{ANNEXES}

\section{Protocole de transcription}

Les phénomènes suivants seront ignorés durant la transcription :

- Les bruits perturbateurs (sirènes, sonneries, aboiements etc.).

- Les éternuements, rires, pleurs, toux etc.

- Les chevauchements du type backchannel qui correspondent à des mots produits par l'interlocuteur/auditeur pour signifier au locuteur qu'il le suit et qu'il comprend ce qu'il dit.

Une correction de la part du transcripteur est apportée pour les phénomènes similaires à ce qui suit :

- J'sais pas sera retranscrit Je sais pas, mais non Je ne sais pas.

- Les erreurs phonétiques (si le locuteur ne prononce pas correctement un son, il sera quand même retranscrit selon l'orthographe standard, ex : la maison même si le $\mathrm{N}$ a été prononcé).

Aucune majuscule ne sera utilisée sauf si le mot est un nom propre. Un fragment inintelligible sera transcrit par $\mathbf{x x x}$.

En ce qui concerne les amorces et les faux départs, une esperluette sera apposée en début du fragment : une mai mai maison sera transcrit une \&mai \&mai maison 
Balises et traitements spécifiques à CLAN :

\begin{tabular}{|c|c|c|}
\hline $\begin{array}{l}\text { Les acronymes et } \\
\text { les signes épelés. }\end{array}$ & $\begin{array}{l}\text { Chaque lettre est écrite en majuscule puis } \\
\text { suivie du symbole } \\
\text { Si le sigle est lu, comme par exemple } \\
\text { UNESCO, le mot est écrit en toutes lettres } \\
\text { et avec une majuscule }\end{array}$ & $\begin{array}{l}\text { MIT est transcrit : M_I_T } \\
\text { SNCF } \text { est transcrit : S_N_C_F } \\
\text { Unesco } \text { est transcrit Unesco }\end{array}$ \\
\hline $\begin{array}{l}\text { Les formes } \\
\text { épelées ou lettres } \\
\text { distinctes. }\end{array}$ & Chaque lettre sera suivie de @l & $\begin{array}{l}\text { My name is Smith, } \\
S \text { M I T H est transcrit : my name } \\
\text { is Smith s@l m@l i@l t@l h@l } \\
\text { Je m'appelle Pierre } \\
\text { P IE R R E est transcrit : je m' } \\
\text { appelle Pierre p@l i@l e@l r@l } \\
\text { r@l e@l }\end{array}$ \\
\hline $\begin{array}{l}\text { Les mots } \\
\text { composés. }\end{array}$ & $\begin{array}{l}\text { Le trait d'union est remplacé par le } \\
\text { symbole }+ \text {. } \\
\text { Les locutions seront traitées comme le } \\
\text { sont les mots composés. }\end{array}$ & $\begin{array}{l}\text { Week-end donne week+end } \\
\text { Grand-père donne grand+père. } \\
\text { In fact donne in+fact } \\
\text { En effet donne en+effet. }\end{array}$ \\
\hline $\begin{array}{l}\text { Les titres et les } \\
\text { nombres. }\end{array}$ & $\begin{array}{l}\text { Les titres et les nombres sont transcrits en } \\
\text { toutes lettres. }\end{array}$ & $\begin{array}{l}\text { Dr donne doctor ou docteur. } \\
7: 15 \text { donne seven fifteen. } \\
2008 \text { donne deux mille huit. }\end{array}$ \\
\hline $\begin{array}{l}\text { Les mots en } \\
\text { langue étrangère }\end{array}$ & $\begin{array}{l}\text { Le mot est suivi de @s:xx } \\
\mathrm{xx} \text { étant le bigraphe représentant la } \\
\text { langue: en pour anglais, ar arabe, zh } \\
\text { chinois mandarin, fr français et es } \\
\text { espagnol. }\end{array}$ & $\begin{array}{l}\text { My father has been muté donne } \\
\text { my father has been muté@s:fra. } \\
\text { J'ai passé ma driving license } \\
\text { donne j' ai passé ma } \\
\text { driving@s:eng license@s:eng }\end{array}$ \\
\hline $\begin{array}{l}\text { Les contractions } \\
\text { et assimilations }\end{array}$ & $\begin{array}{l}\text { En anglais, les formes contractées sont } \\
\text { transcrites en respectant l'orthographe } \\
\text { standard, tout comme le génitif saxon. Les } \\
\text { assimilations sont également transcrites } \\
\text { comme telles. } \\
\text { En français, les contractions ou les formes } \\
\text { regroupées sont transcrites comme des } \\
\text { mots séparés par un espace, même si } \\
\text { l'apostrophe est maintenue. }\end{array}$ & $\begin{array}{l}\text { I'm tired, I'll come, I'd like to, } \\
\text { we've got, he doesn't know } \\
\text { seront transcrits tels quels. Ainsi } \\
\text { que I wanna et I gonna. } \\
\text { j'arrive donne j' arrive et il l'a } \\
\text { pris donne il l' a pris. } \\
\text { Est-ce que donne est+ce+que mais } \\
\text { dit-il donnera dit il. }\end{array}$ \\
\hline
\end{tabular}




\begin{tabular}{|c|c|c|}
\hline Les répétitions & $\begin{array}{l}\text { Si la répétition porte sur un mot, il est } \\
\text { suivi de la séquence }\left[\begin{array}{ll}x & a\end{array} \text {, a étant le }\right. \\
\text { nombre de répétitions. } \\
\text { Si la répétition porte sur une suite de } \\
\text { mots, celle-ci est mise entre chevrons et } \\
\text { est suivie de la séquence }[\mathrm{x} \text { a], a étant le } \\
\text { nombre de répétitions. } \\
\text { Les hésitations de type «euh » entre les } \\
\text { répétitions sont ignorées. }\end{array}$ & $\begin{array}{l}\text { I er I am tired donne } \mathrm{I}[\mathrm{x} 2] \text { am } \\
\text { tired. } \\
\text { I want er I want to come donne }<\mathrm{I} \\
\text { want }>[\mathrm{x} 2] \text { to come. } \\
\text { Ce ce euh ce garçon est sympa } \\
\text { donne ce [x } 3] \text { garçon est sympa. } \\
\text { Je crois que euh je crois que je } \\
\text { vais venir donne <je crois que }>[\mathrm{x} \\
2] \text { je vais venir. }\end{array}$ \\
\hline
\end{tabular}

\section{Abridged English version}

The French words "Accentuation" and "intonation" are common elements of prosody as defined for example by Carton (1974), Gadet (1989), Léon (1992), Astésano (2001), but also as defined in a didactic perspective by Lauret (2007) and Intravaia (2000) - the only difference being that Intravaia uses the word stress rather than accentuation. In French, stress turns syllables into prominent syllables and this prominence is fundamental, particularly concerning the learning and teaching of French as a Foreign Language. This stress lengthens the last syllable of the word or of the group. We assume that for didactic purposes, the duration is more important than the other acoustic parameters - fundamental frequency and intensity.

This demarcational stress and the segmentation pause following it mark out groups that are given various names, depending on the perspective chosen. Authors refer to, amongst others, "semantic", "stressed", "rhythmic", "syntactic" or "temporal" group.

According to Carton (1974), "[I]l semble qu'on puisse définir grammaticalement et sémantiquement à la fois des groupes accentuels virtuels, c'est-à-dire susceptibles dans certains contextes de porter l'accent" (It seems possible to define both grammatically and semantically virtual stress groups - that is groups likely to bear stress in certain contexts).

According to Martin (2008), the stress group - that he calls the "prosodic word" contains only one prominent syllable and does not necessarily match with the "meaning group". He considers that there are groups that can bear a stress - but that are not necessarily stressed - in which the words are linked by grammatical dependence. The actual accentuation will then be determined by a rhythmic parameter. This accentuation will take into account Wioland's (1985) rule of the 7 syllables, according to which most stress groups contain from 3 to 7 syllables.

Delais-Roussarie (2000) has the same opinion: according to her, speakers use prosody to emphasize the morphosyntactic structure of their utterance and always respect several fundamental rhythmic principles. Vaissière (2010), when she lists the consensual points on this subject, specifies that the speakers tend to balance the length of successive rhythmic groups.

All these definitions show that there is a close link between prosody and morphosyntax. We can find the same idea, in a teaching perspective close to ours, in Intravaia's work (2007), where he argues that French rhythm takes on an important demarcative function 
(segmental, syntactic). According to him, stresses and pauses set the boundaries of meaning unities and they condition the segmentation of the sentence into semanticosyntactic groups.

As it has close links with morphosyntax, the accent has to be taken into account when teaching or learning French as a Foreign Language. This article is based on an interlanguage corpus database under the CLAN software. The database consists in interviews of second language learners. The present paper aims at showing the links between prosodic and morphosyntactic errors. The analysis - which is not contrastive and as a consequence will not consider the interferences between the source and the target languages - will correlate the absence of stress on the last syllable of the group and/or the wrong segmentation of the speech chain with the morphosyntactic errors.

The corpus under scrutiny is composed of forty recorded interviews. It is actually a subcorpus, the full corpus consisting for each learner in a 20- to 30-minute interview, a 3minute reading and a written paper. Twenty-five interviews were conducted by native speakers and the other fifteen were conducted by advanced learners. The interviewed students were at the B2 or C1 level in the CECRL. Twelve of them were Arabic speakers, eight were English speakers, thirteen were Spanish speakers and seven were Chinese speakers. The corpus, intended for distribution to the scientific community, is currently being finalized but can already be exploited. The interlanguage corpus database is set up by the students of the Master 1 Linguistics and didactics of languages, English as a Foreign Language and French as a Foreign Language at Rennes 2 University, as part of their training in interlanguage analysis.

The selected parts in .wav, extracted from the 16-hour corpus, were analysed under PRAAT (Boersma \& Weenink 2001). This analysis aimed at confirming the auditory impression concerning the presence of lengthened syllables and segmentation pauses.

The first results of the analysis show on the one hand that exchanges such as greetings and questions rephrasing reveal that non-native speakers use the prosodic marks in a way that is different from the native speakers. The results show on the other hand that there are links between morphosyntactic and prosodic errors, but that those links are not systematic. Finally, they show how and under what conditions enumeration and repetition match the expected prosody.

First of all, during the moments of interlocution, the restatements show that in order to make their utterance more understandable the experienced speakers (native or nonnative) increase the number and/or the duration of the pauses. The moments of greetings show that the non-native speakers hardly lengthen the syllable supposed to be longer, if at all - the last syllable of "bonjour" ${ }^{2}$ has the same length as the first one, and can even be shorter -, and that they adopt a segmentation in which the orthographic word is the basic prosodic unit, at the expense of the rhythmic group. In the group "Bonjour + Name", the non-native speakers separate the words by a pause, a thing native speakers never do. Concerning morphology, we can note that the determiner is missing, which shows that perception and expression are based on lexical words. In the interlocution, the nouns phonetically invariable lexical words in the corpus- are given an unwanted selfreferential value when the students take them up again alone, without a determiner. This is what happens for example in: 
eng_az_gr_92_f_13

*ENQ: les \# c'est comment \# décris euh \# est+ce+que tu peux décrire ?

*FLE: hum \# des [x 2] grandes maisons \# \&an anciennes \# pour les euh \# les rois \# ou les [x 2] royal.

*ENQ: les châteaux.

*FLE: châteaux. ${ }^{10}$

In all the examples in which the nouns are used without a determiner, the primacy is given to the lexical words and not to the noun phrase. This absence, however, can be heard in the prosody: the pause and/or the "euh" indicate that the group is not complete and that the speaker can probably feel it. This is what we note for example in:

zho_yi_wa_93_f_13

[...] les Français \# aiment des choses comme \# [0,29 ms] fromage \# [892 ms] beurre. 11

Concerning the syntax, we note the absence of the subordinating conjunction "que" in structures such as "je crois que" (I think that). The comment clause "je crois" (I think), when it is inserted in the middle or at the end of the utterance, is always used as expected and correctly segmented by two pauses. On the contrary, when "je crois" is used without a subordinator, it is almost never followed by a pause. This is what we observe for example in:

zho_yi_wa_93_f_13

je crois c'est intéressant \# et les questions \# je crois \# c'est beaucoup plus grand \# que ce+que j' ai prévu. ${ }^{12}$

We can see that syntax and prosody actually correlate: when both are correct they reinforce each other, but when the syntax is wrong, the segmentation is absent and thus does not compensate for the missing subordinator.

But we can also find prosodic successes in this corpus, when structures are reiterated. When the speaker enumerates French or gallicized toponyms, the stressed syllable is clearly lengthened. We can hear it for example in:

ara_di_ma_83_f_09

*ENQ: tu as visité quelles régions?

*FLE: euh Paris \# Dor $\underline{\text { dogne }}$ \# Versailles \# et \# Normandie. ${ }^{13}$

spa_ar_ca_73_f_10

*ENQ: qu'avez vous fait \# pendant les vacances?

*FLE: ah \# je suis allée \# à Paris \# je suis allée \# à \# Amsterdam \# je suis allée aussi \# à Rome \# oui \# et bon \# c'est tout. ${ }^{14}$

Besides, in all the enumerations, lengthening and segmentation are performed as expected: same-function unities follow one another, which makes it easier for the speaker to adopt and repeat a prosodic model, as for example in: 
spa_al_ga_79_h_12

on a trouvé [...] des choses à faire \# des choses à \# enregistrer \# des choses à con naître \#

et \# relationner \# avec $\mathrm{l}^{\prime}$ informatique $[. . .]^{15}$

When repetition confirms a lexical and/or a morphological choice, it is "naturally" through prosody (or would it rather be the morphology that confirms the rhythm?). In this situation, the speaker's prosody will tend to respect the principles of isochronism, eurhythmy and progression. This is the case for example in this enumeration in which the speaker talks about the French language. The groups: "c'est romantique / c'est musi $\underline{\text { cal }}$ [...] / c'est la langue de l'amour" respectively

spa_ar_ca_73_f_10

mais \# le français \# je l'aimais bien \# ah \# parce+que \# on disait \# ah c'est

romantique \# c'est musical \#.

tu sais \# il+y+a beaucoup de clichés \# par+rapport+à la langue

française \#.

et \# c'est la langue de l'amour \# des choses comme+ça \# n'est+ce+pas. ${ }^{16}$

The learning and teaching of French as a Foreign Language can benefit from all those observations, and we should always bear in mind that phonetics and morphosyntax converge. As a matter of fact, it seems necessary to explain that the language is a system in which the basic unity - for morphology and prosody - is not the orthographic word but a group that is composed, if we take the example of the noun group, at the very least of a determiner and a noun. It also seems necessary to show that comment clauses and subordinate clauses, for example, are not interchangeable, neither considering their syntax nor their prosody. This corpus confirms that there is a major difference between native and non-native speakers: the native speakers' prosody takes over from an approximative or "ordinary" syntax. This is not the case for non-native speakers. And this is another reason for those learning and teaching French as a Foreign Language not to forget how close and strong the links connecting those linguistic parameters are.

\section{NOTES}

1. CIL : pour Corpus Interlangue.

2. CLAN : pour "Computerized Language ANalysis », outil weblex pour l'annotation de données phonologiques et morphologiques.

3. Conventions de transcription :

- «'» placé devant la syllabe accentuée signale une accent tonique (pour les exemples en espagnol) et un accent de groupe (pour les exemples en français) ;

- «" » signale un accent d'emphase ;

- le soulignement d'une syllabe indique son allongement ;

- «\# » signale une pause.

4. C.I.R.E.F.E. : Centre International Rennais d'Etudes du Français pour Etrangers (Rennes 2).

5. LIDILE, université Rennes $2:$ L'unité de recherche LIDILE a été habilitée Equipe d'accueil (EA 3874) en 2004. Transdisciplinaire et inter-universitaire, elle intègre des enseignants-chercheurs des deux universités de Rennes. Elle est actuellement dirigée par Marie-Claude LE BOT. 
Les recherches s'articulent autour de trois axes :

- des travaux en linguistique appliquée menés au sein de l'axe ELIA (Etudes en Linguistique Appliquées), dirigé par Elisabeth RICHARD et Betina SCHNABEL.

- des travaux en recherche-action développés par DILEM (Didactique des langues et Multimédias), axe dirigé par Nicole CLOAREC et Aura DUFFE.

- des travaux en traduction spécialisée inscrits dans l'axe TRASILT (Traduction spécialisée, ingénierie linguistique et terminologie), dirigé par Katell HERNANDEZ-MORIN.

LIDILE sollicite une démarche multidisciplinaire autour du thème fédérateur «Analyse de l'activité langagière ", en favorisant la pluralité des regards, des compétences et des réflexions, et en mettant en synergie les recherches développées.

www.univ-rennes2.fr/lidile

6. Le corpus est construit sous la responsabilité de Najib ARBACH, doctorant à LIDILE.

ARBACH N. \& ALI S. (2013) Aspects théoriques et méthodologiques de la représentativité des corpus, in CORELA - Numéros thématiques | Statut et utilisation des corpus en linguistique, Montpellier.

ARBACH N. (2010) L'outil CLAN pour la constitution et l'analyse d'un corpus d'apprenants en FLE, in Publication des communications de la journée de présentation des travaux des doctorants et jeunes docteurs en Linguistique française, Brescia, Italie.

7. «Praat est un logiciel Open Source de phonétique permettant l'analyse de toute donnée sonore" (définition sur le site de Framasoft: [http://www.framasoft.net/article3362.html]) programmé par Paul Boersma et David Weenink, Institute of Phonetic Sciences, University of Amsterdam. Le logiciel est téléchargeable sur : [http://www.praat.org] La version utilisée est la version 5.4.

8. C'est d'ailleurs bien sur ce principe que s'appuie par exemple Monique Callamand dans ses exercices systématiques de prononciation de L'Intonation expressive (1973).

9. Hello.

10. Surveyor: The... how is it, describe er... can you describe?

Student: hum... some (x2) big houses an... ancient for the er... the kings or the (x2) royal

Surveyor: the castles.

Student : castles.

11. Student: [...] French people like things like cheese butter (those two nouns require the article in French, which is not the case in English).

12. Student: I think it is interesting and the questions I think it is much bigger than what I had expected. (the conjunction that is compulsary in French after think).

13. Surveyor: Which parts of the country did you visit?

Student : Er... Paris, Dordogne, Versailles and Normandie.

14. Surveyor: What did you do during your holidays?

Student: Ah... I went to Paris, I went to Amsterdam, I also went to Rome, yes, and that's all.

15. We found things to do, things to record, things to know and linked with computer science.

16. "But French I liked it because, ah, because we used to say, ah, it is romantic, it is musical. You know, there are many clichés concerning French. And it is the language of love, and things like that, isn't it. 


\section{RÉSUMÉS}

En français, l'accent dit tonique fait de la syllabe qui le porte une syllabe proéminente, primordiale en particulier pour l'enseignement-apprentissage du FLE. Non distinctif, cet accent est démarcatif, c'est-à-dire qu'il signale le découpage de la parole en groupes de mots. Ces groupes sont déterminés par le sens, la syntaxe et le rythme, et leur frontière droite est marquée par l'accent - et en particulier par l'allongement de la dernière syllabe pleine - et souvent, une pause de segmentation. Posant que morphosyntaxe et prosodie sont liées, cet article analyse une base de données CIL sous CLAN, constituée d'interviews de locuteurs francophones non natifs, de façon à déterminer si ou dans quelle mesure les erreurs prosodiques sont concomitantes et symptomatiques d'erreurs morphosyntaxiques. L'écoute des entretiens et la vérification au moyen de Praat montrent chez de nombreux locuteurs une prosodie non attendue qui isole des mots graphiques. L'analyse morphologique met en évidence que des pauses apparaissent là où un déterminant était nécessaire. Concernant la syntaxe en revanche, l'absence du subordonnant «que» n'est pas compensée prosodiquement. Il apparaît enfin que diverses structures réitératives entraînent la prosodie attendue, respectant même les principes d'eurythmie et de progression.

See annex

\section{INDEX}

Keywords : French as a foreign language, database, prosody, morphology, syntax

Mots-clés : français langue étrangère, base CIL, prosodie, morphologie, syntaxe

\section{AUTEUR}

\section{MARIE-FRANÇOISE BOURVON}

Université Rennes 2 - LIDILE EA 3874

marie-francoise.bourvon@uhb.fr 